Musées, Patrimoine et Culture scientifiques et techniques

$120 \mid 2008$

novembre - décembre 2008

\title{
Des livrets pour accompagner les enfants dans les musées
}

Audrey Van Dorpe et Lucie Scamps

\section{OpenEdition}

\section{Journals}

Édition électronique

URL : https://journals.openedition.org/ocim/313

DOI : 10.4000/ocim.313

ISSN : 2108-646X

Éditeur

OCIM

Édition imprimée

Date de publication : 1 novembre 2008

Pagination : 4-10

ISSN : 0994-1908

Référence électronique

Audrey Van Dorpe et Lucie Scamps, « Des livrets pour accompagner les enfants dans les musées », La Lettre de l'OCIM [En ligne], 120 | 2008, mis en ligne le 19 janvier 2011, consulté le 07 décembre 2022 URL : http://journals.openedition.org/ocim/313 ; DOI : https://doi.org/10.4000/ocim.313 
futur visiteur. De questionnaires factuels, les documents d'accompagnement à la visite sont devenus des invitations à l'observation, au questionnement personnel, voire à la créativité et au développement de la sensibilité.

Mis à disposition gratuitement dans la plupart des cas, les livrets pour enfants ne sont pas nécessairement coûteux à concevoir et réaliser. Toutes les structures culturelles ont les moyens d'offrir aux enfants ne serait-ce qu'un feuillet pour accompagner leur visite. Dans certaines institutions, chaque tranche d'âge a son livret, les documents pour les groupes scolaires sont parfois différents, ils sont à utiliser dans le cadre d'une visite guidée ou en autonomie avec l'enseignant.

Comment s'effectuent ces choix ? Comment concevoir un document d'accompagnement à la visite pour enfants ? Les publications et les études qui répondent à ces questions sont rares. Quelques enquêtes ponctuelles ont permis d'évaluer des livrets d'aide à la visite, mais chaque document étant de conception différente, il est difficile de généraliser toutes leurs conclusions. Nous souhaitons amorcer ici une réflexion quant à la conception de ces livrets et leur utilité. Dans quels buts sont-ils conçus ? Comment atteindre des objectifs pédagogiques définis ?

Une étude formelle d'un corpus de documents d'accompagnement à la visite nous a servi de support afin de proposer des conseils pratiques de conception et des hypothèses de recherches futures ${ }^{(1)}$.

\section{Séduire}

Un des rôles premiers des documents d'accompagnement à la visite est de séduire le visiteur afin qu'il en fasse l'usage. Les livrets séduisent tout d'abord par leur présence dans la structure, car ils répondent à une demande d'information qui émane des visiteurs et offrent une alternative à la visite guidée en accompagnant les enfants dans les expositions. La seconde mission de séduction de ces livrets est de procurer du plaisir à l'enfant visiteur en lui donnant une mission à remplir de manière ludique. Le but est que l'enfant ressorte du musée en ayant compris ce qu'il a vu sans s'être ennuyé.

Après la visite, l'enfant emmène son livret à la maison, en souvenir. Ce document produit par le musée est un élément de communication. Le concepteur doit donc penser à rendre la structure identifiable sur le document, à l'aide du nom ou du logo, mais aussi en insérant quelques informations pratiques pouvant inciter une éventuelle venue ultérieure : horaires et jours d'ouverture, prix d'entrée. Ces informations doivent cependant rester discrètes et ne pas interférer avec le propos du livret.

Afin d'inciter l'enfant à utiliser le livret, le concepteur possède plusieurs options complémentaires qui rendront le document plus attrayant. Le titre est un premier moyen de séduire l'enfant. Il est évident que Parcours-jeu aura potentiellement moins de succès que Mène l'enquête au musée ! L'intitulé doit être amusant, ludique, jouer sur l'imaginaire de l'enfant, lui donner un rôle. Cependant, le concepteur doit veiller à ne pas perdre de vue le sujet de l'exposition qui doit prédominer. C'est pour cela qu'offrir une récompense à l'enfant est déconseillé. Dans ce cas, il se dépêchera de remplir son livret, la compétition prendra le pas sur la visite. De plus, cela l'encourage à répondre juste, sous la menace de ne pas avoir sa récompense, ce qui biaise le rôle du musée qui sera dès lors assimilé à l'école et ses bons points.

L'enfant est plus à même d'utiliser, de lire et de garder un document d'accompagnement à la visite plaisant à feuilleter. C'est pourquoi le concepteur doit porter une attention particulière au graphisme, aux illustrations, à la mise en forme du livret. Varier les polices de caractères et les couleurs peut également rendre la lecture plus agréable, à condition de le faire en quantité raisonnable faute de quoi, on risque de rendre le document illisible. Ces outils vont permettre de hiérarchiser le contenu du livret afin que l'enfant se repère plus facilement. Il est bien d'intégrer également des illustrations, que ce soit des photographies ou des dessins. Toutefois proposer des jeux qui puissent être réalisés uniquement à l'aide de ces illustrations risque de détourner l'enfant de l'exposition : il se servira de la reproduction pour répondre aux questions, sans regarder l'objet.

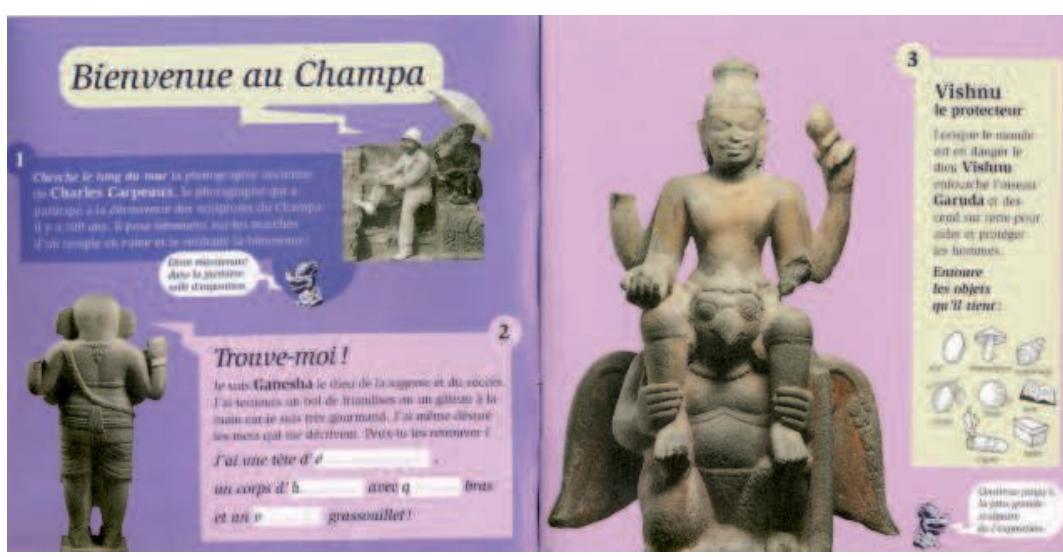

Le graphisme est un élément de séduction. (๑) Musée national des Arts asiatiques Guimet 


\section{Quelques conseils protiques}

- Mettre les livrets à disposition à l'accueil du musée, à bonne hauteur afin qu'ils soient visibles par les enfants. Mettre également les livrets en téléchargement sur le site Internet du musée.

- Si le livret nécessite d'écrire, distribuer à l'accueil un crayon et un support à l'enfant, tout en le sensibilisant, lui et son accompagnateur, sur la prudence dont il faut faire preuve avec un crayon près des collections.

- Choisir un format pratique, facile à manipuler par l'enfant.

- La mise en page facilite l'appréhension du document, les consignes sont clairement énoncées, les caractères sont suffisamment grands pour être lus dans les salles sombres.

Par ailleurs, l'intégration de petits dessins humoristiques qui ponctuent le livret peut apporter une pointe d'humour, toujours la bienvenue chez les enfants : il faut cependant veiller à ce que ces dessins ne véhiculent pas de fausses informations, notamment des stéréotypes. Les enfants ne perçoivent pas facilement la différence entre les éléments que l'on veut dénoncer et ceux que l'on veut leur apprendre, surtout si cela est fait par le biais de l'humour, ils ne retiendront que ce qui les a amusés.

Les documents d'accompagnement à la visite doivent également séduire les accompagnateurs des enfants, qui inciteront ces derniers à leur utilisation. Les parents apprécieront sans doute de pouvoir visiter le musée en famille, de voir leurs enfants découvrir l'exposition seuls, mais également de pouvoir partager leur visite en utilisant le livret ensemble. La réalisation d'un petit livret à destination des parents qui contiendrait le plan spatial et thématique de l'exposition, les réponses aux jeux du livret pour les enfants et des conseils pour accompagner son enfant dans une exposition est un plus. Ce livret peut inclure des activités à faire en famille dans l'exposition et des questions que les parents peuvent poser aux enfants pour stimuler leur attention.

\section{Former les jeunes visiteurs}

Un des objectifs des documents d'accompagnement à la visite est de permettre à l'enfant de découvrir une exposition par lui-même. En effet, un livret bien conçu rend l'enfant autonome dans l'exposition, il peut parcourir les salles seul, possède les informations dont il a besoin, et réalise des activités et des jeux qui aiguisent son regard et sa réflexion. Un document d'accompagnement à la visite est un moyen de former les visiteurs. Un document ludique révèle à l'enfant l'intérêt d'une exposition, donne une image distrayante du musée. Il remplit une part de ses objectifs s'il permet à l'enfant d'apprécier sa visite et l'incite à revenir au musée.

L'enfant aime se sentir considéré comme un adulte et se sentira valorisé de pouvoir faire ses propres découvertes. Il n'est pas pour autant seul avec son livret dans l'exposition, il est accompagné soit d'un enseignant, ou d'un médiateur ou de sa famille. Le rôle de cet accompagnateur est très important dans la visite de l'enfant. Il l'oriente, l'aide parfois, le renseigne, et s'amuse avec lui. Un échange a lieu entre l'enfant et l'adulte, le partage d'une expérience et ceci en partie grâce au livret. En effet, le parent s'intéresse à ce que fait son enfant, l'enfant souhaite évoquer ses découvertes et peut même expliquer certains points de l'exposition. Le document fait naître facilement une convivialité, l'enfant et l'adulte passent un bon moment. Il reste cependant un élément à prendre en compte, le parcours de visite de l'enfant ne sera pas le même que celui de l'adulte, le temps de visite non plus. Il faut donc tenir compte de ce paramètre lors de la conception d'un document, choisir attentivement les œuvres ou objets qui y figureront. C'est pour cela que nous proposons de concevoir deux documents complémentaires, un pour l'enfant et l'autre pour l'accompagnateur, ce qui permet de garder un lien tout au long de la visite entre ces deux individus.

Si les échanges entre les visiteurs sont importants, le document doit favoriser le plus possible l'autonomie de l'enfant, qui en retirera une grande satisfaction. Afin qu'il se sente prêt à visiter seul ou presque, il faut lui faciliter le parcours et l'accompagner au maximum. Le concepteur peut ainsi insérer un plan de l'exposition avec éventuellement un repère signifiant l'emplacement des œuvres ou objets reproduits dans le livret. Des repères visiblement reconnaissables par les enfants peuvent également être insérés dans le parcours de visite, afin de faciliter la déambulation et l'orientation du jeune visiteur dans l'exposition.

L'introduction d'un personnage-guide, présenté au début du livret, est un moyen efficace pour donner des consignes de visite. Ces consignes sont importantes pour que l'enfant fasse la transition entre l'extérieur du musée et l'exposition. Elles doivent être relativement courtes pour s'assurer que l'enfant les lisent, car elles sont primordiales dans des livrets annonçant 


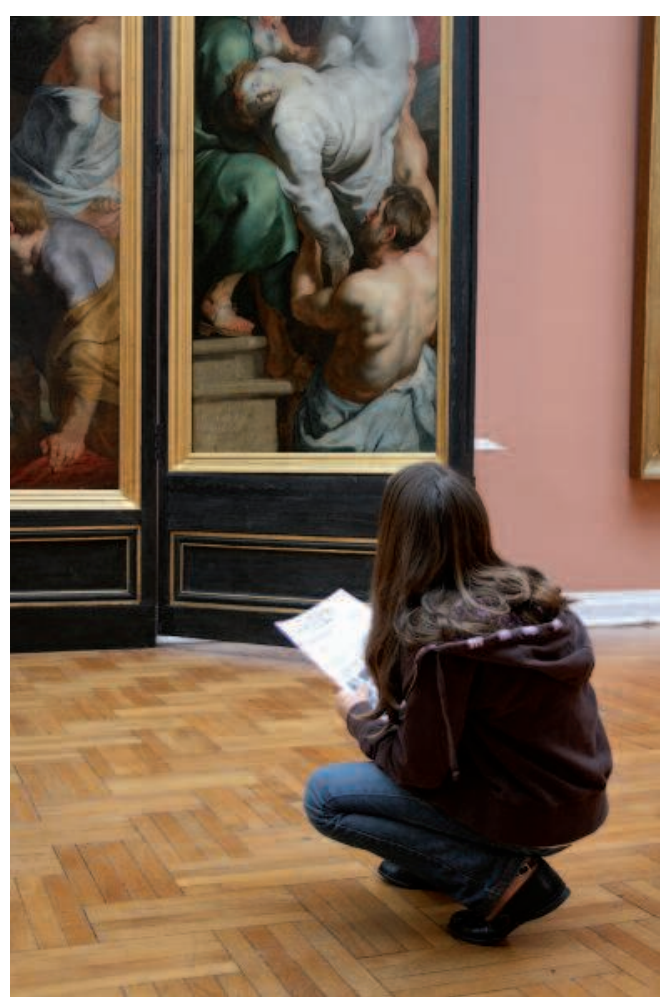

De nombreux livrets guident I'observation. (c) Musée des Beaux-Arts de Valenciennes/Isabelle Nouhaud

une enquête dont elles expliquent le déroulement. Le personnage-guide doit être en rapport avec l'exposition, donne des consignes à l'enfant sur ses déplacements par exemple, guide le regard et les déplacements de l'enfant, lui donne les consignes pour réaliser les différentes activités et l'informe sur l'exposition en s'adressant à lui comme à un ami.

Les couleurs employées et la typographie sont importantes afin de hiérarchiser le contenu, mais il est aussi intéressant d'intégrer des pictogrammes qui signalent des actions particulières proposées à l'enfant : écrire, regarder, se déplacer... Ils sont également utiles pour les enfants ne sachant pas lire, ils seront ainsi plus autonomes. Il est possible d'insérer dans le document d'aide à la visite un glossaire des notions importantes, spécifiques ou compliquées utilisées dans le livret et dans l'exposition afin de favoriser l'autonomie de l'enfant. Il ne faut jamais perdre de vue les caractéristiques de son public et ainsi adapter la difficulté du livret à l'âge et aux aptitudes de l'enfant. Ce glossaire peut être situé à la fin du livret ou au fil des pages dans de petits encarts. Le dernier élément très important pour la réussite de la visite de l'enfant est de lui donner les solutions aux jeux et questions posées dans le livret, pour qu'il ne soit pas frustré de ne pas savoir si ce qu'il a fait est juste ou non. Ces solutions peuvent se trouver dans le livret de l'enfant, dans celui de l'accompagnateur ou à

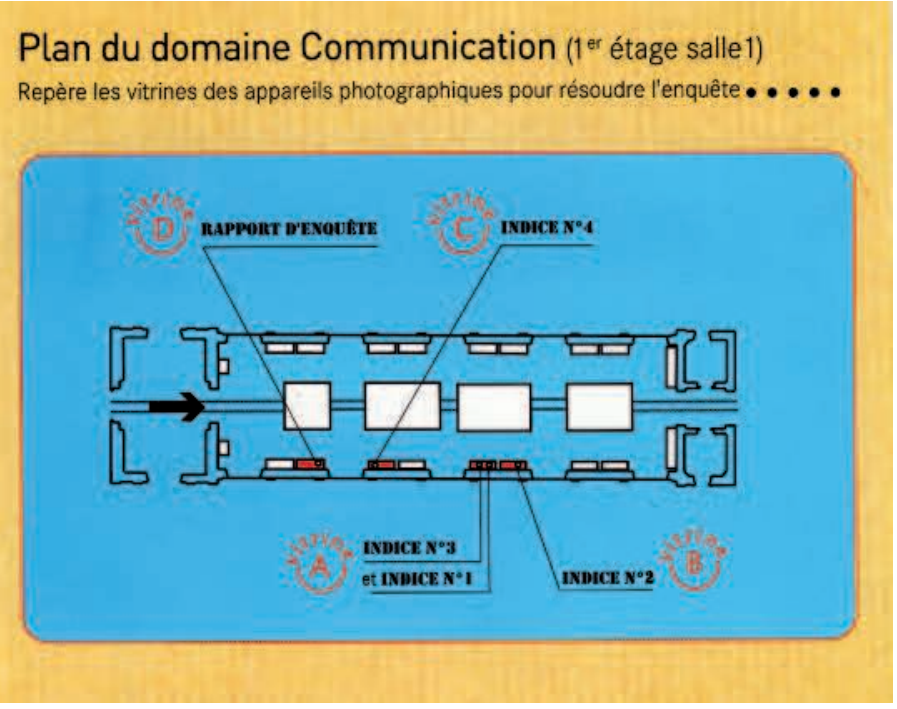

Le plan est un relais essentiel entre le livret et l'exposition. (C) Musée des Arts et Métiers

la fin de la visite. Cependant, nous déconseillons de donner une note à l'enfant. L'idéal est de proposer des activités qui ne demandent pas de réponse précise, juste ou fausse, afin que le livret ne soit pas assimilé à une interrogation scolaire, qui sousentend qu'il y a de bons et de mauvais visiteurs.

Si le livret est un moyen efficace d'aider l'enfant à être autonome dans une exposition, il n'en reste pas moins que le choix des œuvres et objets présentés dans le livret et sur lesquels l'enfant s'arrêtera est primordial. Il faut penser que si les éléments exposés sont trop éloignés les uns des autres, mal indiqués, obligeant l'enfant à déambuler dans tous les sens, celui-ci abandonnera vite son livret. Si le livret impose un parcours à l'enfant, il faut veiller à ce qu'il ne soit pas trop différent de celui de son accompagnateur, mais aussi faire attention à ce que l'effet «parcours-livret » (2) ne canalise pas trop l'enfant. Celui-ci doit pouvoir appréhender l'exposition et pas uniquement les œuvres ou objets présentés par le document d'accompagnement à la visite.

Il est donc simple de rendre le jeune visiteur autonome par les moyens précédemment énumérés, mais ces derniers doivent rester en accord avec les objectifs pédagogiques choisis. Le livret doit être un moyen au service d'objectifs bien définis, et non pas une fin en soi. 


\section{Comment accompagner un enfant dans sa visite ?}

Les documents conçus par les structures muséales et patrimoniales répondent à des objectifs variés, dont le principal consiste à permettre à l'enfant de s'approprier l'exposition. Le livret accompagne, c'est-à-dire guide et encourage cette activité intellectuelle et émotionnelle. Il permet également de la rendre concrète, notamment par un biais dynamique, actif et ludique. D'ailleurs, la mémorisation est beaucoup plus importante lors d'une activité ou d'une expérimentation que lors d'une visite « passive ». Les structures culturelles conçoivent des livrets pour les enfants dans le but de faciliter leur accès au savoir, de canaliser leur attention durant la visite, ou plus simplement d'éveiller leur curiosité et de les familiariser avec l'institution. La conception d'un document d'accompagnement à la visite doit être motivée par un ou plusieurs objectifs pédagogiques. S'agit-il d'encourager l'enfant à l'observation ? De lui apporter des notions sur un sujet ? De stimuler un questionnement personnel ?

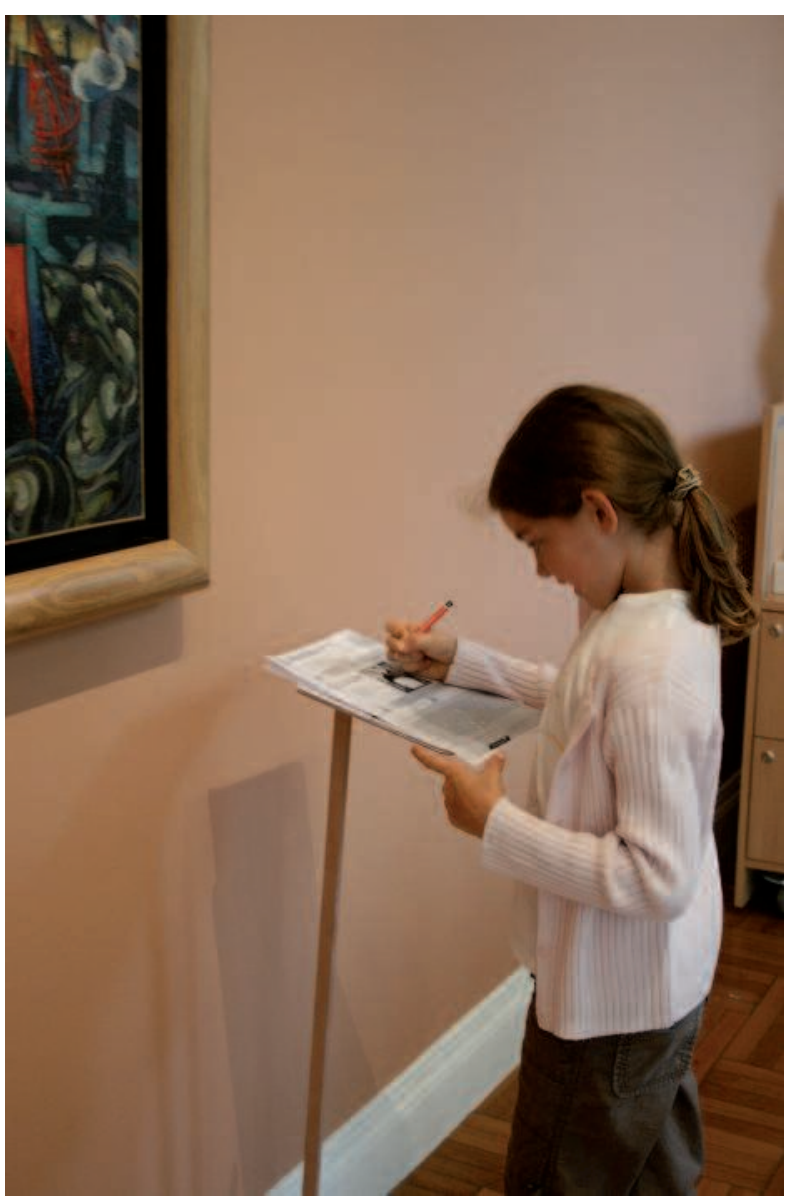

Ne pas oublier des supports pour écrire, sinon les enfants trouveront un endroit qui n'est pas forcément adapté, comme ici les cartels.

○) Musée des Beaux-Arts de Valenciennes/Isabelle Nouhaud
La définition de ces objectifs, tout comme le choix des types d'activités pour les mettre en œuvre, se fait en fonction de lâge des enfants. Il est nécessaire de cibler le public auquel s'adressera le livret, afin d'en connaître le stade de développement cognitif, les représentations et les connaissances préalables. Les activités et le discours doivent être adaptés à l'âge des enfants, les questions qui font appel à la culture générale sont déconseillées car trop proches du modèle scolaire. Le livret doit avant tout permettre l'exploration de l'exposition, dont le contenu sert de base aux activités proposées à l'enfant.

L'interaction de l'enfant avec son livret dynamise nécessairement sa visite. Néanmoins, textes ou jeux, les moyens d'accompagner l'enfant ont des qualités variées. Il semble bon de stimuler l'enfant par différentes activités qui le rendent actif tout au long de sa visite. Visiter avec un livret c'est explorer, découvrir et pour cela observer, associer, lire, jouer, imaginer... Certains documents proposent à l'enfant de jouer un rôle, d'être acteur d'une mise en scène. Ce ressort ludique permet à l'enfant de s'approprier le sujet de l'exposition en s'identifiant à un personnage, issu du thème de l'exposition ou bien à un détective ou un explorateur par exemple. Une intrigue peut inciter l'enfant à poursuivre sa visite jusqu'à la fin. Toutefois, il faut veiller à ce que la part de scénario et de jeu permette bien la découverte de l'exposition, et ne prenne pas le pas sur cette dernière.

Le jeu est tout à fait indiqué pour guider l'enfant dans sa visite, ce moyen de médiation se distingue nettement du mode d'apprentissage scolaire, il est pour cela apprécié des enfants. Mais encore une fois, le jeu doit être utilisé à bon escient, il ne faut pas que l'enfant se presse de « gagner » ou cherche à dévier les activités pour trouver plus facilement une réponse qui lui permettrait de progresser dans le jeu. Nous distinguons plusieurs types de jeux qui sont à choisir en fonction des objectifs pédagogiques définis par la structure conceptrice du livret. Dans la plupart des musées, les jeux qui stimulent l'observation sont fondamentaux : comparer une illustration et un objet exposé ou repérer les détails d'une œuvre sont des activités qui aiguisent le regard. Les informations que le concepteur du livret souhaite dispenser à l'enfant peuvent prendre la forme de jeux de mots, de questionnaires à choix multiples, de rébus. Ceux-ci mobilisent les connaissances de l'enfant sous une forme ludique. De nombreux livrets proposent des jeux de reproduction qui demandent à l'enfant de mimer, faire à la manière de, voire de copier une œuvre ou de reproduire un objet. Sans 
l'intervention d'un médiateur pour, par exemple, expliquer la technique à employer, ce type de jeux est à éviter car il apporte peu à la découverte de l'enfant. À l'inverse, faire appel à l'imagination de l'enfant en lui suggérant de décorer ou de dessiner permet un investissement affectif, profitable à la visite tant que la consigne reste en lien étroit avec l'exposition. Ce type d'activités permet de faire une pause dans la visite et introduit un moment de plus grande détente, cependant il faut être attentif à la durée de la visite.

Jeux et activités prennent très souvent la forme de questions. Trois formes principales de questions sont identifiées :

- les questions conceptuelles font référence aux connaissances de l'enfant pour lui permettre de concevoir de nouvelles idées en relation avec le sujet de l'exposition ;

- les questions factuelles sont fermées, elles prennent pour source les informations dispensées par l'exposition ;

- les questions contextuelles demandent de synthétiser et d'évaluer des concepts, elles engagent à la créativité (3).

Enfin, les questions qui convoquent le vécu de l'enfant stimulent l'expression de ses impressions, ce qui favorise tout à fait son appropriation de l'exposition.

Le texte dans les livrets pour enfants a différentes fonctions : donner les consignes, informer sur le sujet de l'exposition ou être le support même d'une

Maintenant, observons ensemble comment Ferdinand Bol, un élève doué de Rembrandt, raconte cette histoire.

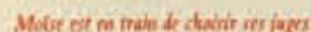
Co mone poraceble: à un courensement

dive mariagr

a une messe

I wi adabement de chevalier

Retrouve sur le tableau les personnages de l'histoire :

- Moïse - Jethro

- Le(s) juge(s)

Quels moyens utilise le peintre pour mettre Moïse en valeur? poor mentrer la richesse de Moise parce quik n'suait pes de daaseas powr rendre la sethe mains séricusr pour $u$ faine plaisir Essaie de retrouver ces détails dans le tableau. Attention, it $y$ a un intrus II

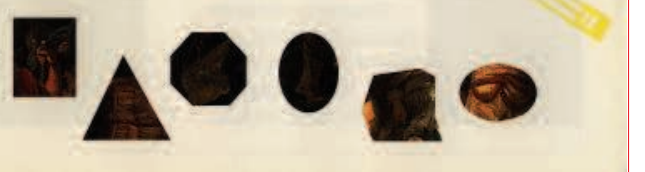

Mille et une façons de répondre aux questions (c) Musée des Beaux-Arts de Dijon activité, s'il s'agit d'associer une source littéraire avec l'œuvre qu'elle a inspirée par exemple. La quantité de texte est un choix que fait le concepteur en fonction de ses objectifs pédagogiques, et de l'âge des enfants. Pour les plus jeunes qui maîtrisent mal la lecture, les activités utilisant le dessin ou l'association sont à privilégier. La rédaction des textes doit être claire et simple, au présent de narration, le vocabulaire doit être adapté sans pour autant devenir simpliste. Souligner ou mettre en italique les termes et notions importantes participe de la hiérarchisation du texte. Les informations complémentaires peuvent faire l'objet d'encarts, tout comme les mots les plus complexes sont indexés dans un lexique. Tutoyer l'enfant, utiliser des formes exclamatives, des points de suspension rend le texte plus dynamique. La présence, la quantité et le contenu des textes doivent être mûrement réfléchis lors de la conception du livret.

Le livret crée l'interactivité entre l'enfant et l'exposition, pour cela il est demandé à l'enfant d'être actif. Les actions qui lui sont proposées doivent être variées : observer, associer, lire, décrire ; mais également les moyens de restituer ses réponses : dessiner, cocher, relier, entourer, écrire. Si varier les actions dynamise le document, il faut néanmoins veiller à ne pas perturber l'enfant par des consignes trop changeantes. Un autre point est à prendre en considération lors de la conception d'un livret : la durée des activités. Les enfants ne sont pas dans des conditions leur permettant de formuler à l'écrit de longues réponses ou de faire plusieurs dessins. L'appréhension de l'exposition reste le principal but de la visite, et l'enfant ne doit pas garder les yeux rivés sur son livret. D'ailleurs, si le concepteur demande trop souvent à l'enfant d'écrire des réponses, il court le risque que celui-ci abandonne son livret. Concernant les questionnaires à choix multiples, s'ils permettent de répondre simplement en cochant, ils présentent à l'enfant des réponses erronées. Ils impliquent donc qu'il y ait une correction du livret afin que l'enfant n'assimile pas de mauvaises informations. La durée d'attention des enfants dépend à la fois du type d'activités proposées et de leur âge. L'utilisation d'un document d'accompagnement semble augmenter le temps de visite des enfants, cependant il est préférable d'envisager une visite d'environ une heure et que le livret ne dépasse pas une vingtaine de pages. Le concepteur peut inviter l'enfant à poursuivre les activités chez lui ou en classe, si leur lien avec l'exposition le permet. 
Texte, questions et jeux sont généralement illustrés. Il est important que les illustrations relient les activités et l'exposition pour l'appropriation de celle-ci, mais également pour que l'enfant parte du musée avec des images associées à sa visite. Les enfants mémorisent facilement les illustrations et elles comblent en quelque sorte la frustration de ne pas pouvoir toucher les collections. Enfin, photographies des objets dans un autre contexte ou portraits des artistes, cartes et frises chronologiques donnent des repères spatio-temporels essentiels pour les enfants.

Concevoir un livret d'accompagnement à la visite est un réel projet pour une structure muséale. Il ne s'agit pas simplement de poser des questions auxquelles l'enfant trouve les réponses en lisant les cartels. Certes, apprendre à lire les textes de l'exposition participe de la formation de l'enfant visiteur, mais un livret est également l'occasion de former son regard et son jugement. C'est pourquoi le concepteur doit éviter d'induire un parcours déterminé, il est nécessaire que l'enfant ait une part de décision dans son exploration. Cette liberté offre à l'enfant la possibilité de s'intéresser à ce qui attire son attention, le livret est là pour l'aider dans son observation, ses interrogations et facilite l'expression de ses impressions.

Un document d'aide à la visite a indubitablement un impact sur l'enfant, c'est un outil au fort potentiel. Il est indispensable que les objectifs qui président à la conception d'un livret soient correctement clarifiés. Des tests permettent de s'assurer de la validité de ces objectifs, tout comme les évaluations sommatives permettent d'apprécier l'efficacité du livret.

\section{Noles}

(1) Ces réflexions sur la conception de livrets pour enfants sont issues d'une étude menée par nos soins dans le cadre universitaire en février 2008. Il s'agissait de l'analyse formelle d'un corpus de 47 documents donnés, vendus, ou bien diffusés sur Internet par des musées et sites patrimoniaux, et destinés aux enfants de 2 à 12 ans en visite dans le cadre familial ou scolaire.

Nous avons confronté les documents récoltés à l'aide de 39 critères d'analyse précis, répartis en cinq thèmes : «informations générales », «pour accompagner les enfants », « images et mise en forme », « actions mobilisées par l'enfant » et « jeux ». L'étude et l'observation formelle de ces documents ont abouti à des propositions de conseils de conception à destination des professionnels des musées.

(2) Gottesdiener, H. et Vilatte, J.-C. Évaluation d'un outil pédagogique dans le cadre d'une visite familiale. Direction des Musées de France, 2000. (3) Allard, M. et Boucher, S. Éduquer au musée : un modèle théorique de pédagogie muséale. Montréal : HMH, 1998, 207 p.

\section{Bibliographie}

Allard, M. et Boucher, S. Éduquer au musée : un modèle théorique de pédagogie muséale. Montréal : HMH, 1998, 207 p.

Girardet, S. et Merleau-Ponty, C. Portes ouvertes : les enfants : accueillir les enfants dans un musée ou une exposition. Paris : Musée en Herbe ; Dijon : OCIM, 1994, 31 p.

Gottesdiener, H. et Vilatte, J.-C. Évaluation d'un outil pédagogique dans le cadre d'une visite familiale. Direction des Musées de France, 2000.

Guichard, J. Démarche pédagogique et autonomie de l'enfant dans une exposition scientifique, Aster, $n^{\circ}$, 1989, pp. 17-42.

Guidon, C. Les Livrets pour enfants dans les musées d'ethnologie et de civilisation. Mémoire d'étude de l'École du Louvre sous la direction de Claire Merleau-Ponty et Carole Millière, 2003, 58 p.

Hall, N. (ed.) Writing and designing interpretive materials for children. Manchester : Center for Environmental Interpretation, 1984, 77 p.

Merleau-Ponty, C. Les enfants dans les musées : encore un petit effort, la Lettre de l'OCIM, nº72, 2000, pp. 16-17.

Tuzi, D. Quand les enfants «font » de la Préhistoire, la Lettre de l'OCIM, n 113,2007 , pp. 26-33. 